\title{
Hypomagnesaemia causing myopathy and hypocalcaemia in an alcoholic
}

\author{
H.S. Pall, ${ }^{1}$ A.C. Williams, ${ }^{1}$ D.A. Heath, ${ }^{2}$ M. Sheppard,${ }^{2}$ and R. Wilson. ${ }^{3}$ \\ ${ }^{1}$ Department of Neurology, ${ }^{2}$ Department of Medicine, Queen Elizabeth Hospital, Birmingham, B15 2TH and \\ ${ }^{3}$ Department of Medicine, Solihull Hospital, Lode Lane, Solihull, B91 2JL, UK.
}

\begin{abstract}
Summary: A well-nourished alcoholic patient developed a subacute myopathy which responded rapidly to correction of severe hypomagnesaemia. The finding of profound hypocalcaemia prompted the measurement of serum magnesium. Magnesium deficiency should be looked for in any alcoholic patient with a myopathy as the prognosis seems better than in many other forms of alcoholic myopathy. Correction of the magnesium deficiency corrects the hypocalcaemia without the need for calcium supplementation.
\end{abstract}

\section{Introduction}

The cause of subacute alcohol associated myopathy is not always clear. Hypokalaemia has been implicated ${ }^{1}$ but clinical response to correction of hypokalaemia does not always occur. We describe a hypomagnesaemic hypocalcaemic normokalaemic patient with such a myopathy that responded well to magnesium supplementation.

\section{Case report}

A 65 year old well-nourished woman presented with a 6 week history of muscle cramps and arm and leg weakness predominantly affecting proximal musculature. She had a long history of excessive alcohol intake and had suffered a short episode of similar weakness 1 year previously which had resolved spontaneously. She took frusemide $\mathbf{4 0} \mathrm{mg}$ a day for intermittent dependant oedema and was taking potassium supplements. Proximal muscle weakness was pronounced in both arms and legs and there was some muscle twitching but no fasciculation. Chvostek's sign was positive but Trousseau's sign was negative. Although deep tendon reflexes were absent there were no other features of peripheral neuropathy.

Investigations revealed the following serum abnormalities (normal ranges in brackets): aspartate transaminase $79 \mathrm{IU} / 1$ (normal 5-30), albumin $30 \mathrm{~g} / 1$ (3846), bilirubin $27 \mu \mathrm{mol} / 1(3-21)$, calcium $1.35 \mathrm{mmol} / 1$ (2.10-2.55) and magnesium $0.20 \mathrm{mmol} / 1(0.70-0.95)$. Alkaline phosphatase remained between 200-220 IU/1 (70-350) throughout the illness. Serum potassium and

Correspondence: H.S. Pall, M.B., M.R.C.P.

Accepted: 11 February 1987 creatine kinase levels were also normal. Electromyography (EMG) showed myopathic change and sensory action potentials were normal. Muscle biopsy (vastus lateralis) showed scattered atrophic muscle fibres with no group atrophy and no evidence of myositis.

Serum magnesium was measured within hours of admission to hospital after serum calcium had been found to be profoundly low. The hypocalcaemia was thought to be secondary to the hypomagnesaemia ${ }^{2}$ and attributable predominantly to alcoholism and partly diuretic therapy. Replacement of the magnesium deficit and withdrawal of alcohol and diuretics resulted in a gradual return of serum calcium concentration to normal (Figure 1). Neuromuscular irritability resolved over the first 2 days; the proximal muscle weakness taking about 2 weeks to recover. Deep tendon reflexes remained absent. Following treatment the serum calcium was $2.2 \mathrm{mmol} / 1$, serum magnesium $0.92 \mathrm{mmol} / 1$ and serum phosphate $1.09 \mathrm{mmol} / 1$ (normal $0.8-1.4$ ).

\section{Discussion}

Hypomagnesaemia may cause hypocalcaemia and under these conditions attempts at correcting the hypocalcaemia with calcium supplements and/or vitamin $D$, even in massive doses, are futile. ${ }^{2}$ The treatment of this form of hypocalcaemia requires administration of magnesium rather than calcium - a point of management that makes hypomagnesaemia a very important differential diagnosis in any patient with unexplained severe hypocalcaemia. Recognition

(C) The Fellowship of Postgraduate Medicine, 1987 


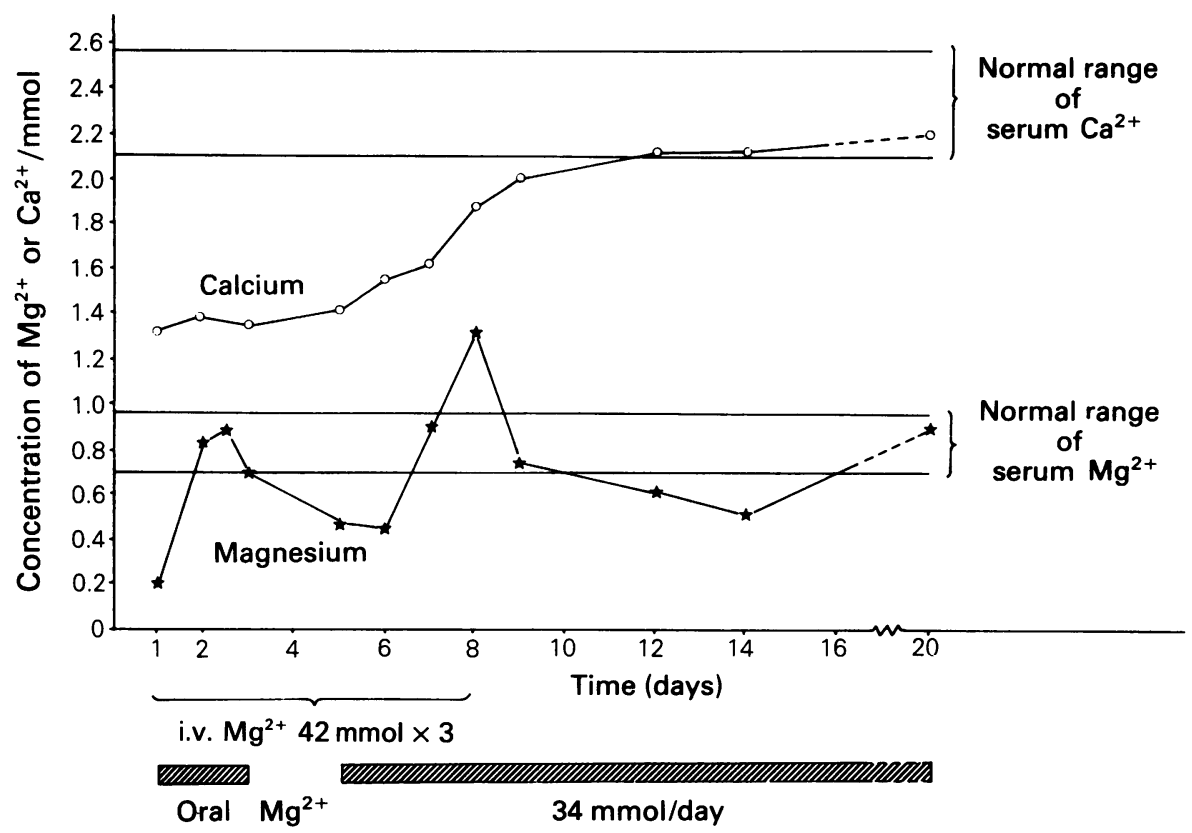

Figure 1 Changes in serum magnesium and calcium with magnesium supplementation.

of this fact avoids unnecessary and futile administration of calcium supplements and vitamin $D$. The pathogenesis of this secondary hypocalcaemia has been reviewed by Berkelhammer ${ }^{3}$ and Rude $^{4}$ and briefly involves both a decrease in parathyroid hormone (PTH) secretion and a peripheral resistance to PTH action, although impaired 25-hydroxylation of vitamin D may also be involved. ${ }^{5}$ Correction of the magnesium deficit results in resolution of the hypocalcaemia over a few days as illustrated by our patient (Figure 1). We also demonstrate that serum calcium concentrations do not closely follow acute changes in serum magnesium concentrations suggesting that intracellular magnesium levels are more important in influencing calcium homeostasis. Our patient was given more than $750 \mathrm{mmol}$ of magnesium ions over 3 weeks to correct the hypomagnesaemia. This emphasizes the fact that, as with potassium, magnesium is mainly an intracellular element. Consequently patients may have significant depletion of body magnesium even when the serum magnesium concentration is normal. ${ }^{5}$

Hypomagnesaemia is well recognized as a complication of alcoholism and is thought to be secondary to decreased intake, reduced absorption and excessive renal losses. ${ }^{6}$ Magnesium depletion is not generally recognized as a possible cause of alcoholic myopathy. Cases of alcoholic myopathy have been attributed to hypokalaemia even when there has been associated hypomagnesaemia and when clinical improvement has followed treatment with both potassium a $9 \overrightarrow{0}$ magnesium supplements. ${ }^{7}$ There is some evidence thit muscle may be at particular risk of magnesifang depletion. For instance Lim \& $\mathrm{Jacob}^{8}$ showed reduct-乏 tion in muscle magnesium in alcoholics who haof normal bone magnesium levels and McIntyre hase shown the same to occur in magnesium deficiency dued to severe malabsorption. Experimental depletion of magnesium in animals causes alteration in muscle resting transmembrane electrical potential ${ }^{10}$ and even muscle necrosis. " In normal human volunteers such depletion causes myopathic changes on EMG which resolve with magnesium repletion. ${ }^{2}$ Fonseca \& Havard $^{12}$ have reported a patient with cardiac failure secondary to hypomagnesaemia and in the absence of 3 . arrhythmias. Cardiac muscle with its similarities to skeletal muscle may have expressed a myopathy in a马 similar manner to our patient's skeletal muscle myopathy. However, the patient they reported was so severely malnourished that various other nutritionato deficiencies may have contributed to the clinical picture.

Our patient shows that subacute alcoholion myopathy may be due to magnesium deficiency and may show good response to magnesium repletion. $\omega$ Although significant magnesium depletion may occur? in the absence of hypomagnesaemia or hypocal $\frac{\text { 은 }}{2}$ caemia, we suggest that measurement of serum mag $\frac{\$}{\text { is }}$ nesium concentration should form part of the inves:tigation of every alcoholic patient with a myopathy 


\section{References}

1. Martin, J.B., Eckel, R.E., Craig, J.W. et al. Hypokalaemic myopathy in chronic alcoholism. Neurology 1971, 21: $1160-1168$.

2. Shils, M.E. Experimental human magnesium depletion. Medicine (Baltimore) 1969, 48: 61-85.

3. Berkelhammer, C. \& Bear, R.A. A clinical approach to common electrolyte problems: 4. Hypomagnesaemia. Can Med Assoc J 1985, 132: 360-368.

4. Rude, R.K., Oldham, S.B. \& Singer, F.R. Functional hypoparathyroidism and parathyroid hormone endorgan resistance in human magnesium deficiency. Clin Endocrinol 1976, 5: 209-224.

5. Reddy, V. \& Sivakumar, B. Magnesium-dependent vitamin D-resistant rickets. Lancet 1974, i: 963-965.

6. Haller, R.G. \& Knochel, J.P. Skeletal muscle disease in alcoholism. Med Clin North Am 1984, 68(1): 91-103.
7. Rubinstein, A.E. \& Wainapel, S.F. Acute hypokalaemic myopathy in alcoholism: a clinical entity. Arch Neurol 1977, 34: 553-555.

8. Lim, P. \& Jacob, E. Magnesium status of alcoholic patients. Metabolism 1972, 21: 1045-1051.

9. McIntyre, I., Hanna, S., Booth, C.C. \& Read, A.E. Intracellular magnesium deficiency in man. Clin Sci 1961, 20: 297-305.

10. Cronin, R.E. \& Knochel, J.P. Magnesium deficiency. Adv Intern Med 1983, 28: 509-532.

11. Haggtveit, H.A. Myopathy in experimental magnesium deficiency. Ann NY Acad Sci 1969, 162: 758-765.

12. Fonseca, V. \& Havard, C.W.H. Electrolyte disturbances and cardiac failure with hypomagnesaemia in anorexia nervosa. Br Med J 1985, 291: 1680-1682. 